\title{
Determinación De Áreas De Inundación En El Municipio De Chia- Colombia Mediante Hec-Ras En La Cuenca Baja Del Río Frio
}

\author{
Carlos Peña-Guzmán, MD \\ Universidad Autónoma de Colombia, \\ Departamento de Ingeniería Ambiental, Bogotá Colombia \\ Natalia Alvarino \\ Constanza Mora \\ Universidad Manuela Beltrán, \\ Espacialización de Evaluación de Impacto Ambiental \\ Duvan Mesa, MD \\ Universidad Santo Tomas de Colombia, \\ Departamento de Ingeniería Ambiental
}

doi: 10.19044/esj.2016.v12n5p386 URL:http://dx.doi.org/10.19044/esj.2016.v12n5p386

\begin{abstract}
The phenomenon La Niña caused flooding in Colombia during 2010 and 2011, especially in the upper basin of the Bogota River, this natural risk has produced major property damage and a decrease in socio-economic conditions in major urban and rural centers. For that reason, this paper presents a determination of the floodplain of the lower basin of the Rio Frio in the urban area of Chia Colombia by HEC RAS. The model allowed to determine the maximum water levels reached on the floodplain of the Rio Frio as it passes through the urban area of Chia, for return periods of rainfall of 2.33, 5, 10, 25, 50 and 100 years. Finally recommendations for the planning of the municipality in terms of these areas found are made.
\end{abstract}

Keywords: Floodplain, HEC-RAS, haydraulic simulation

\section{Resumen}

El fenómeno de La Niña causó inundaciones en Colombia durante el año 2010 y 2011, sobre todo en la cuenca alta del río Bogotá, este riesgo natural ha producido los mayores daños en propiedades y una disminución en las condiciones socioeconómica de los principales centros urbanos y rurales. Por esa razón el siguiente artículo presenta una determinación de las áreas de inundación de la cuenca baja del Río Frío en el área urbana del 
municipio de Chía Colombia mediante HEC- RAS. El modelo permitió determinar los niveles máximos de agua alcanzados sobre las áreas de inundación del Río Frío a su paso por el casco urbano del municipio de Chía, para periodos de retorno de lluvias de 2.33, 5, 10, 25, 50 y 100 años. Por último se hacen recomendaciones para la planificación de la municipalidad en función de estas áreas encontradas.

Palabras clave: Área de inundación, HEC-RAS, simulación hidráulica

\section{Introducción:}

Las inundaciones presentadas durante la temporada de lluvias en los años 2010 y 2011 en Colombia se presentó por el fenómeno de La Niña y en particular en la cuenca alta del río Bogotá. Estas inundaciones a lo largo de la historia han producido pérdidas en las propiedades públicas y privadas y la han generado disminución en la calidad socioeconómica de los centros urbanos y rurales (Sedano-Cruz, et al., 2013).

Por consiguiente en los últimos años se ha ampliado el interés en el estudio de los riesgos naturales a los que están sujetos las zonas urbanas, para lo cual el uso de modelos matemáticos es una de las herramientas más comunes y más precisas para evaluar escenarios de inundación (Brunner, 1995, Stewardson and Gippe, 2003, Triviño and Ortiz, 2004), ya que permiten determinar y análizar el riesgo al que están sometidos las poblaciones (Büchele et al., 2013).

En Colombia, estos modelos están siendo utilizados hace unos quince años aproximadamente, inicialmente como una herramienta para evaluar el flujo de tránsito en ríos y arroyos, para el diseño de estructuras, adicionalmente se han utilizado para definir las áreas susceptibles a inundaciones, apoyados por otros análisis espacial como los sistemas de información geográfica (CORANTIOQUIA, 2005).

De acuerdo a lo anterior, el presente artículo presenta la detreminación de áreas de inundación sobre la cuenca media del Río Frío para periodos de retorno de lluvias de 2.33, 5, 10, 25, 50 y 100 años, adicionalmente se realizan recomendaciones para la planificación de la municipalidad en función de estas áreas encontradas.

\section{Ubicación geográfica:}

La Subcuenca del río Frío se encuentra ubicada al norte de la Cuenca del Río Bogotá, tiene una extensión aproximada de $202 \mathrm{~km}^{2}$, el cauce principal tiene una longitud de $58.9 \mathrm{Km}$ y se localiza en altitudes entre los 2600 y $3700 \mathrm{msnm}$, se caracteriza por presentar topográficamente relieves montañosos en un porcentaje superior al de $60 \%$ del área total, tiene una forma asimétrica, cuya geomorfología ha sido afectada fundamentalmente 
por condiciones geológicas de formación de la sabana del río Bogotá, por estructuras, por la dinámica del drenaje, por los procesos erosivos y por la intervención antrópica.

La distribución de precipitación a lo largo del año es de tipo bimodal, el primer período de lluvias se presenta entre los meses de marzo, abril y mayo, siendo este ultimo el mes más lluvioso, con un valor cercano a los $100 \mathrm{~mm}$. En el segundo semestre se presenta en los meses de octubre y noviembre, siendo octubre el mes con la precipitación mas alta del año, con un valor hasta de $105 \mathrm{~mm}$. El período de estiaje se extiende de diciembre a febrero, observándose a enero como el mes más seco, con un registro del orden de los 28 mm y a mediados del año entre los meses de junio a septiembre, observándose que agosto es el más seco, con un registro de $50 \mathrm{~mm}$. El valor total anual promedio está entre 770 a 885 mm.

\section{Materiales y métodos:}

Inicialmente se recopiló y analizó toda la información disponible relacionada con la zona de influencia del área de estudio a nivel regional, principalmente usos del suelo y usos del recurso hídrico, cartografía consistente en planchas a escala 1:100.000 y 1:750.000, precipitaciones, propiedades físicas del suelos, características hidráulicas y climatológicas. Adicionalmente se llevaron a cabo visitas de campo sobre puntos específicos relacionados con el cauce del río Frío y cruces de puentes vehiculares, información que permitió, verificar las condiciones actuales de la corriente. Esta inspección de campo permitió observar la condición de algunos aspectos morfológicos, patrón de drenaje y dinámica fluvial del río Frío en la zona urbana especialmente en los sitios de cruce importantes.

Para el análisis de los caudales de creciente se empleó la información (caudales y niveles de láminas de agua) de tres estaciones seleccionadas Santa Pozo Hondo, Puente Calamar y Puente la Virginia, el cualculo se llevo a cabo por el modelo de lluvia-escorrentía, para este método se calcularon las curvas IDF para los períodos de retorno de 2.33, 5, 10, 25, 50 y 100 años, para lo cual se emplearon las estaciones de precipitación Almaviva y Alco.

Estos resultados permitieron llevar a cabo los estudios hidráulicos mediante el programa HEC-RAS, los cuales estuvieron orientados a la estimación de la altura máxima del nivel del agua y la velocidad del flujo durante los eventos de crecida máxima.

\section{Resultados:}

La cabecera de la cuenca presenta un cauce con un canal principal central y zonas de planicie lateral dedicadas al uso como pastos para ganadería y algunas parcelas de pancoger de maíz, papa y hortalizas. El cauce es poco profundo con sección en forma de V, con frecuentes saltos en 
roca y algunos deslizamientos de menor tamaño. Debido a la presencia de zona de cobertura, unido a las características orográficas y de clima, el río presenta un caudal perenne. En esta zona el caudal medio fluye por el canal central y una vez ocurren eventos de avenida el cauce no tiene la capacidad permitiéndose el desborde lateral hacia la planicie.

A partir de aproximadamente la cota $2550 \mathrm{msnm}$, se inicia la urbanización de la cuenca, con pequeños núcleos de viviendas y urbanizaciones; en este sector el río continua en cauce natural. Dada la configuración del cauce las crecientes se confinan prácticamente dentro del cauce principal de la quebrada.

En esta zona al final del tramo ocurre inundación de las zonas cercanas al cauce, a pesar de que se encuentran ocupados por vías y áreas libres. Algunos sectores tienen construido un jarillón sobre las márgenes que evita el desbordamiento hacia la urbanización, a pesar de que este se encuentra por debajo del nivel de terreno original.

La zona más baja se caracteriza por la construcción de un jarillón de protección lateral, especialmente sobre la margen izquierda, que protege de desbordes la zona más urbanizada del municipio. Bajo esta condición geométrica, el cauce actual del río permite el tránsito, de todas las crecientes, solo que hacia la margen derecha se presentan desbordes a partir de la creciente de 25 años.

La representación espacial de los eventos de amenaza por inundación para cada evento de crecida hidrológica en el Río Frío, es decir con período de retorno de 2.33, 5, 10, 25, 50 y 100 años se puede ver en la Figura 1.

Finalmente se planteó la generación de zonas de amenaza para el desarrollo urbanístico y terrotorial del municipio, para lo cual se encontró:

\section{Zonas de Amenaza ALTA (Zonas Restringidas)}

Comprenden los eventos de crecientes repentinos de carácter extremo o histórico con probables recurrencias de 25 años o superiores, cuyos caudales superan la capacidad de conducción del cauce. 

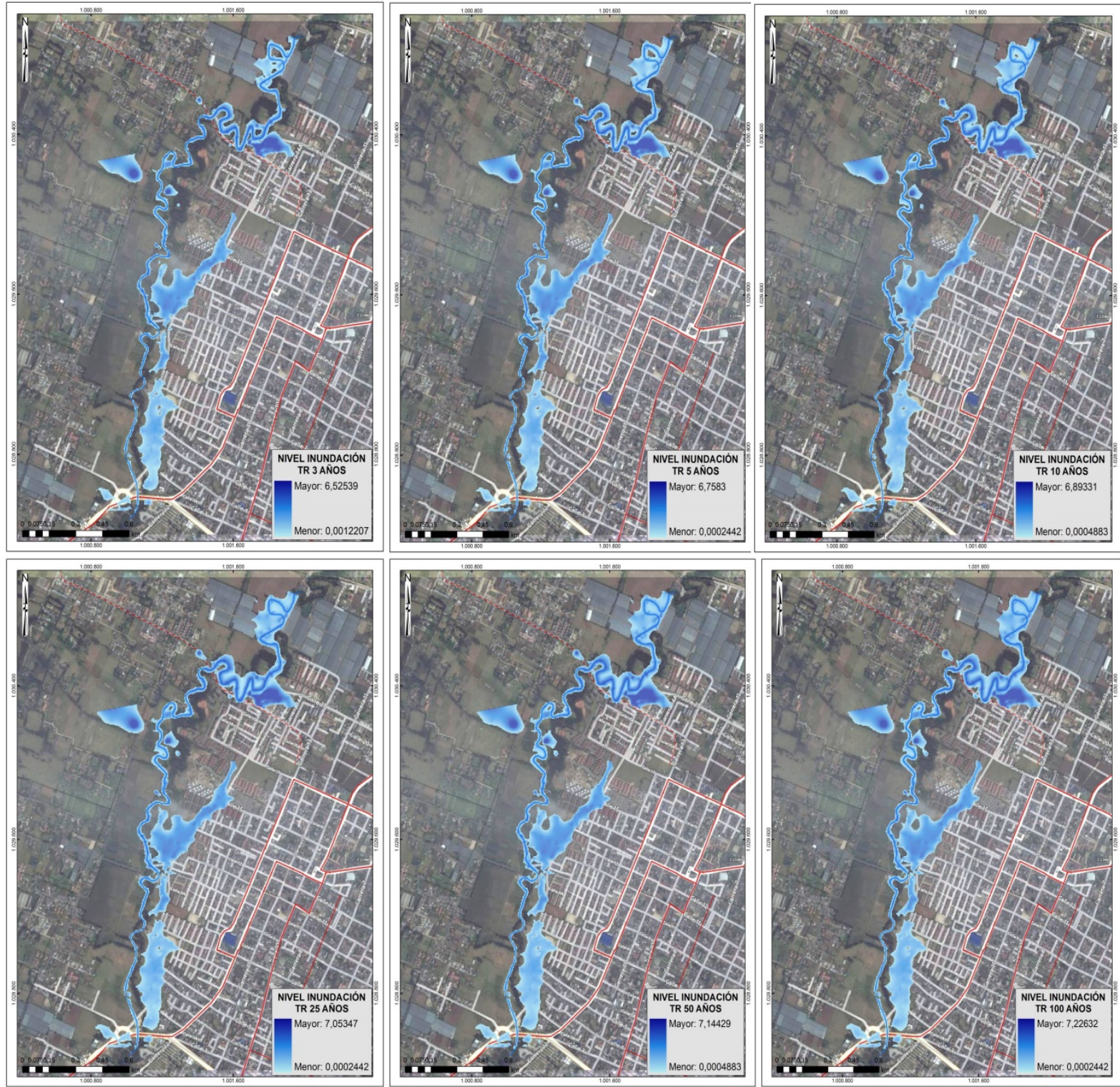

Figura 1. Mapas de amenaza por inundación en la cuenca baja del río Frío, correspondiente a una creciente con tiempos de retorno 2.33, 5, 10, 25, 50 y 100 años

En estos sectores las obras de infraestructura urbana deben ocupar lugares apropiados, con baja densidad poblacional, distribución abierta y límites de propiedades que no signifiquen una variación sustancial de la dinámica fluvial (interceptores de aguas negras, revestimientos, muros, protecciones marginales, cercos vivos densos, etc.); como así también con el mismo fin los planes de forestación deben considerar los sitios, especies y 
densidades adecuadas.

Esta autorización debe estar supeditada a la toma de medidas previas al evento, entre las que se destaca la evaluación de la amenaza, concientización de la población para posibilitar la autoevacuación, reglamentación de obras civiles, etc.; medidas que juegan un papel fundamental en la mitigación.

\section{Zonas de Amenaza EXTREMA. (Zonas Prohibidas)}

Se incluyen los terrenos incluidos dentro del ámbito del lecho de inundación periódico que se activa con recurrencias probables entre 2,33 a 10 años cuando los caudales superan la capacidad de conducción del lecho ordinario.

Se considera una restricción total de uso, sólo aptas para recreación contemplativa, solarium y recreación diurna. La extrema amenaza definida por la frecuente actividad hídrica y significativos picos de crecida, restringe el uso exclusivamente al dominio público con actividades que no involucren la permanencia continua de personas, áreas de recreación, deportes, etc.). Debe evitarse: (i) la construcción de viviendas, (ii) la instalación de obras de infraestructura en general sin la debida planificación (líneas eléctricas, tuberías de gas, tuberías de acueducto y/o alcantarillado, etc.); especial atención merecen los puentes que puedan obstaculizar el flujo del agua, (iii) la implantación de forestaciones con especies foráneas de gran porte que obstaculicen la dinámica fluvial y representen una situación potencial de amenaza por arrastre y obstrucción de puentes y vados, en particular frente a crecientes extremas, (iv) La construcción de obras destinadas a servicios públicos (hospitales, dispensarios, escuelas, etc.).

\section{Conclusion:}

El uso de modelos hidráulicos permitió encontrar zonas de amenaza de inundaciones a diferentes tiempos de retornos, esto nos muestra como esta herramienta permite la planificación del desarrollo territorial del municipio.

Este uso logró determinar Zonas de Amenaza ALTA, las cuales son aquellas crecientes con probabilidad de recurrencias de 25 años o más. En esta zona no puede permitirse la actividad privada reglamentada, aunque sí obras de infraestructura urbana, las cuales deben ocupar lugares apropiados, que no signifiquen una variación sustancial de la dinámica fluvial (interceptores de aguas negras, revestimientos, muros, protecciones marginales, cercos vivos densos, etc.); como así también con el mismo fin los planes de forestación deben considerar los sitios, especies y densidades adecuadas.

Las zonas de amenaza EXTREMAS, comprende las crecientes que tienen recurrencia anual, hasta de 10 años. El uso que se permitirá será el de recreación contemplativa, solarium y recreación diurna. En esta zona se 
restringe el uso exclusivamente al dominio público con actividades que no involucren la permanencia continua de personas, áreas de recreación, deportes, etc.).

\section{References:}

Brunner W., 1995, HEC-RAS River Analysis System. Hydraulic Reference Manual. Version 1.0.

Büchele B., Kreibich H., Kron A., Thieken A., Ihringer J., Oberle P., Merz B., and Nestmann F., 2006, Flood-risk mapping: contributions towards an enhanced assessment of extreme events and associated risk, Nat Hazards Earth Syst Sci, vol. 6, n. ${ }^{\circ}$ 4, pp. 485-503.

CORANTIOQUIA, 2005, Formulación del Plan de Ordenamiento y Manejo de la cuenca del río Aburrá. Medellín, Medellín, Colombia.

Sedano-Cruz K., Carvajal-Escobar Y., Díaz Á., and Javier Á., 2013, Análisis de aspectos que incrementan el riesgo de inundaciones en Colombia», Rev Luna Azul, n. ${ }^{\circ}$ 37, pp. 219-238.

Stewardson M, and Gippe C. J. 2003, Incorporating flow variability into environmental flow regimes using the flow events method, River Res. Appl., vol. 19, n. ${ }^{0}$ 5-6, pp. 459-472.

Triviño Pérez A. and Ortiz Rojas S., 2004, Methodology for spatially distributed modeling of surface runoff and floodplain's landscape in mediterranean torrential streams, Thecnical report. 